\title{
RESEARCH HIGHLIGHT \\ A machine learning approach to predict response to immunotherapy in type 1 diabetes
}

\author{
Georgia Fousteri ${ }^{1}$, Ely Montee Rodrigues ${ }^{2}$, Gian Maria Giamporcaro ${ }^{2}$ and Marika Falcone $\mathbb{D}^{2}$ \\ Cellular \& Molecular Immunology (2021) 18:515-517; https://doi.org/10.1038/s41423-020-00594-4
}

Type 1 diabetes (T1D) is an autoimmune disease mediated by selfreactive $T$ cells that destroy insulin-producing $\beta$ cells of the pancreatic islets. ${ }^{1}$ The disease is a complex multifactorial disorder resulting from a poorly defined interaction between genetic and environmental factors. Approximately $40-50 \%$ of the disease risk is linked to genes, with the remaining risk arising from environmental factors. HLA genes account for approximately one-half of T1D risk, and the residual risk may be ascribed to more than 40 single-nucleotide polymorphisms (SNPs) in genes outside the HLA region. Among confirmed non-HLA susceptibility genes, the best-characterized polymorphisms are related to INS, PTPN22, IL2RA, IFIH1, and CTLA4. ${ }^{2}$ Islet-specific autoantibodies are a hallmark of T1D and currently the best predictive biomarker of disease progression. ${ }^{3}$ However, the mechanism leading to the loss of humoral tolerance in T1D is not fully understood, with the exception of a few studies showing diminished B cell central tolerance in patients with T1D. ${ }^{4} B$ cells acquire their antibodysecreting function after interaction with a specific subset of CD4 $T$ cells in specialized structures within the $B$ cell follicles of secondary lymphoid organs, known as germinal centers. The CD4 T-cell subset that provides the help necessary for B cells to differentiate into memory $B$ cells and high-affinity antibodyproducing plasma cells is known as follicular helper $\mathrm{T}\left(\mathrm{T}_{\mathrm{FH}}\right)$ cells. ${ }^{5}$

Based on recent understanding of disease pathogenesis, the complex interplay between a multitude of factors (environmental factors, genome, microbiome, and immune alterations) leading to abnormal $T$ and $B$ cell activation and destruction of pancreatic $\beta$ cells varies between individual cases of T1D. Accordingly, T1D should therefore not be considered a single autoimmune disorder but rather a spectrum of pathologies related to different mechanisms, including lack of central and peripheral tolerance, defective immune regulation, abnormal autoimmune activation and altered tissue homeostasis, ultimately resulting in $\beta$ cell autoimmunity. ${ }^{1}$ This concept may have important therapeutic implications, and the failure of clinical trials for T1D may be related to the high degree of heterogeneity in disease pathogenesis. In fact, most of the interventional trials involving immunotherapies in T1D patients, such as the humanized anti-CD3 antibody (teplizumab) and blocking molecules for the costimulatory CD28/CD80 (abatacept) or LFA-3/CD2 (alefacept) pathways, failed to reach their primary endpoint for the tested T1D population while demonstrating good therapeutic response only in a subset of subjects. ${ }^{6-8}$ To date, stratification of T1D patients has been based on age and disease stage, but subgroups of T1D may also be distinguished based on different immunopathogenic mechanisms, i.e., the involvement of a single T/B cell subset and/or immune molecular pathway, leading to $\beta$ cell autoimmunity. Overall, stratification of T1D patients based on disease stage or underlying pathogenic mechanisms may be instrumental for designing better clinical trials, resulting in more significant treatment outcomes.

The recent article by Edner et al. ${ }^{1}$ provides the first evidence that comprehensive immunological profiling permits the stratification of T1D patients into responders/non-responders to immunotherapy based on the identification of the underlying pathogenic mechanisms. In that retrospective study, the authors analyzed blood samples of T1D patients treated with CTLA4-lg (abatacept) and, by using a machine learning approach, identified immunological profiles that stratified the treated T1D patients into responders and non-responders (Fig. 1A). Recent advances in molecular profiling, high-throughput sequencing, and computational efficiency have been largely exploited to develop targeted therapy and immunotherapy for precision medicine in oncology. ${ }^{9}$ For example, cancer genome profiling before immunotherapy treatment has been employed to customize immunotherapy for a given patient, thus significantly improving treatment outcomes in clinical trials with immune checkpoint inhibitors (ICls). ${ }^{9}$ In the paper of Edner et al., this approach was used for the first time to stratify patients and predict therapeutic response in the T1D clinical setting. The abatacept study was chosen since a randomized, double-blind, placebo-controlled trial showed that although adjusted c-peptide levels were 59\% higher in treated T1D patients than in the placebo group, it was clear that some treated subjects benefited more than others, suggesting a possible stratification into responders/nonresponders. In the first part of the study, by using a classical hypothesis-driven approach, the authors demonstrated that abatacept decreases circulating $\mathrm{T}_{\mathrm{FH}}$ cells both at one and twoyear time points (Fig. 1A). Principal component analysis of multiparametric FACS data revealed that the major difference between treated/untreated T1D patients involved specifically $T_{F H}$ cell subpopulations that express CXCR5 and ICOS (ICOS ${ }^{+}$PD- $1^{-} \mathrm{T}_{\mathrm{FH}}$ cells) or were CCR7 ${ }^{\text {Io PD }-1} 1^{+}$CXCR5 ${ }^{+}$(ICOS ${ }^{+}$PD $-1^{+} \mathrm{T}_{\mathrm{FH}}$ cells). Next, the authors used the machine learning algorithm CellCnn, a representation learning approach based on convolutional neural networks, to identify immune cell subsets associated with disease status through a data-driven strategy (Fig. 1A). This analysis further corroborated the manual gating approach and found that both $\mathrm{ICOS}^{+} \mathrm{PD}-1^{+}$and ICOS ${ }^{+} \mathrm{PD}-1^{-} \mathrm{T}_{\mathrm{FH}}$ cells were reduced by abatacept treatment. Previous clinical studies using abatacept in T1D and other autoimmune diseases have indicated that this drug works by interfering with CD28:CD80/CD86 crosstalk, one of the more

\footnotetext{
${ }^{1}$ Regulation of Adaptive immunity Unit, Transplantation and Infectious Diseases, IRCCS San Raffaele Scientific Institute, Milan, Italy and ${ }^{2}$ Experimental Diabetes Unit, Division of Immunology, Transplantation and Infectious Diseases, Diabetes Research Institute (DRI), IRCCS San Raffaele Scientific Institute, Milan, Italy Correspondence: Marika Falcone (falcone.marika@hsr.it)
}

Received: 2 November 2020 Accepted: 7 November 2020

Published online: 14 December 2020 
A

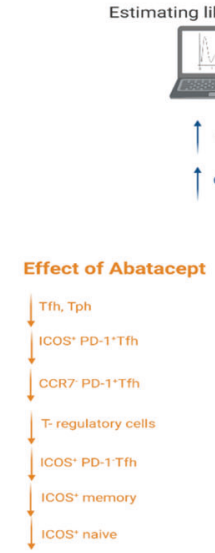

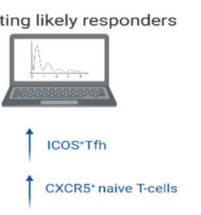
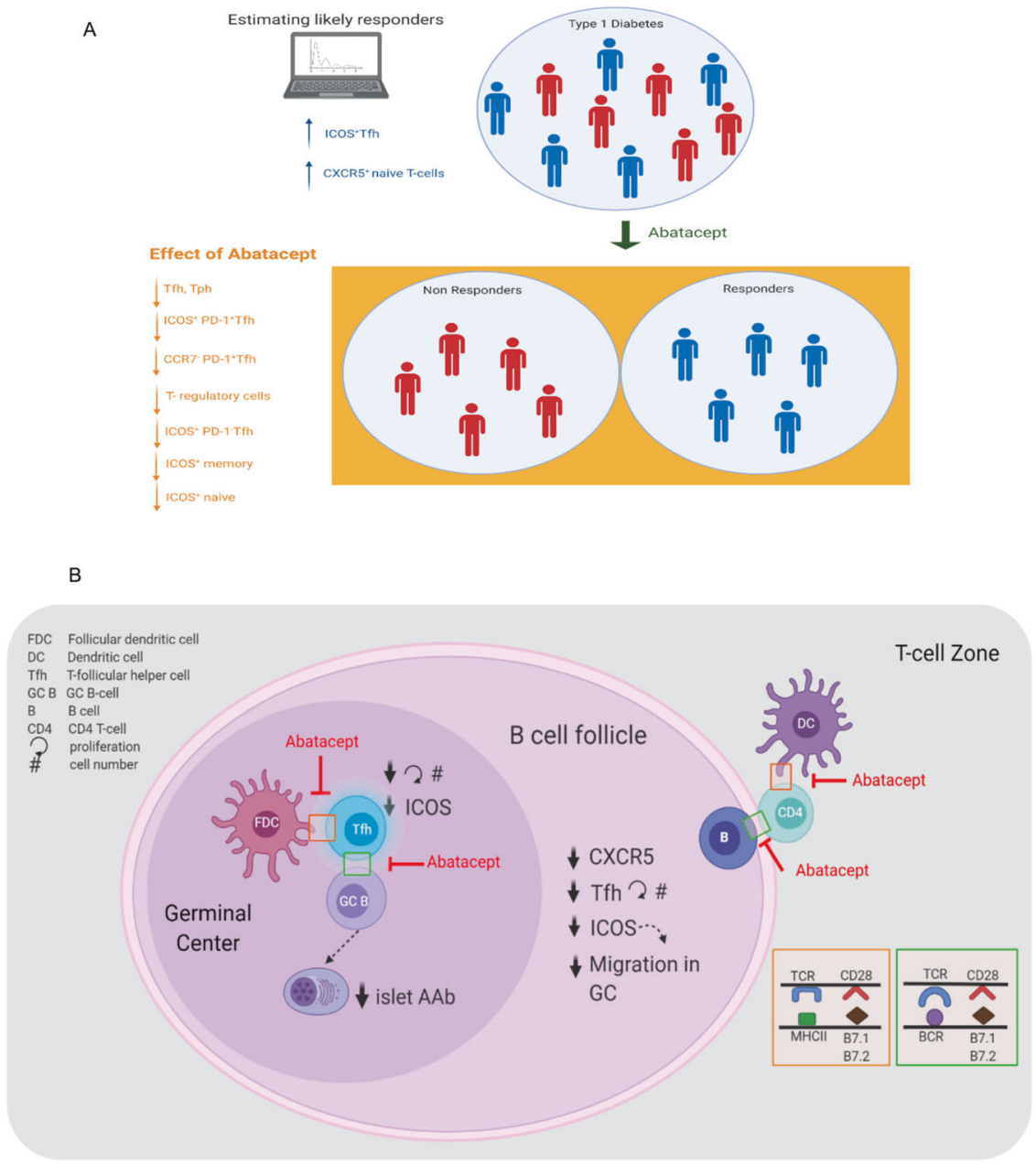

Fig. 1 A Stratification of T1D patients based on immunological profiles. Edner et al. used comprehensive immunological profiling to stratify T1D patients into responders (blue) and non-responders (red). Blood samples of T1D patients treated with CTLA4-lg (abatacept) were analyzed through a machine learning approach that identified immunological profiles (high ICOS ${ }^{+} \mathrm{T}_{\mathrm{FH}}$, high CXCR5 ${ }^{+}$naive $\mathrm{T}$ cells), helping to stratify treated T1D patients into responders and non-responders. Responders were characterized by a reduction in $T_{F H}$ ( $T$ follicular helper), Tph ( $\mathrm{T}$ peripheral helper), ICOS ${ }^{+} \mathrm{PD}-1^{+} \mathrm{T}_{\mathrm{FH}}, \mathrm{ICOS}{ }^{+} \mathrm{PD}-1^{-} \mathrm{T}_{\mathrm{FH}}, \mathrm{T}$ regulatory, and ICOS ${ }^{+}$memory and naive cells. B Possible mechanism of action for CTLA-4lg (abatacept). Deactivation and dedifferentiation of TFH cells may be related to two mechanisms. 1. CTLA-4lg inhibits crosstalk between the costimulatory molecules $C D 28$ and CD80/CD86, altering $\mathrm{T}_{\mathrm{FH}}$ cell differentiation at two "decision" points in the germinal centers of secondary lymphoid organs and the T-cell zone. 2. CTLA-4lg interferes with CD28-CD80/86-mediated activation of CD4 T cells by DCs at the T-cell zone or CD4 T-cell interactions with B cells at the $\mathrm{T}: \mathrm{B}$ cell border, consequently preventing $\mathrm{T}_{\mathrm{FH}}$ cell differentiation, ICOS upregulation, and entry into $B$ cell follicles. Once in the B cell follicle and the germinal center, abatacept neutralizes CD28-CD80/CD86 interaction among $\mathrm{T}_{\mathrm{FH}}$ cells, GC B cells and follicular DCs (FDCs), further weakening $\mathrm{T}_{\mathrm{FH}}$ cell differentiation and proliferation

prominent T-cell costimulatory signals leading to T-cell immunomodulation rather than depletion. ${ }^{10}$ The innovative nature of the work of Edner et al. relates to the observation that in patients with T1D, abatacept targeted a single T-cell subset, namely, circulating $\mathrm{T}_{\mathrm{FH}}$ cells expressing an $\mathrm{ICOS}^{+}$-activated phenotype. This finding suggests that in the T1D cohort of abatacept-responder patients, a mechanism involving $T_{F H}$ cell activation may be responsible for the occurrence of $\beta$-cell autoimmunity. Several studies have also found an increased number of $\mathrm{T}_{\mathrm{FH}}$ cells expressing an activated phenotype $\left(\mathrm{ICOS}^{+} \mathrm{PD}-1^{+}\right)$in patients with T1D and in subjects with a high risk of developing the disease (autoantibody-positive), suggesting a key role for this CD4 T-cell population in islet autoantibody development and disease progression. ${ }^{11}$ Autoantibody development is also controlled by a subset of FoxP3expressing regulatory $T$ cells known as follicular regulatory $\left(T_{F R}\right)$ cells, which were recently found to be altered in T1D. ${ }^{12}$ This suggests that in addition to central tolerance mechanisms, peripheral tolerance mechanisms, particularly $T_{F H}$ and $T_{F R}$ cells, may be compromised in a subgroup of patients who develop T1D.
How does the CTLA-4lg molecule (abatacept) modulate $T_{F H} / T_{F R}$ cell differentiation? CTLA-4lg binds to CD80/CD86 on antigenpresenting cells such as dendritic cells (DCs) and B cells and blocks their interaction with the CD28 molecule, a costimulatory signal that is fundamental for T-cell priming, including $T_{F H}$ cell differentiation and acquisition of an $\mathrm{ICOS}^{+}$phenotype. ${ }^{13}$ Edner et al. speculate that since the Icos gene is CD28 sensitive in other immune cell subsets, it may also regulate ICOS expression on $\mathrm{T}_{\mathrm{FH}}$ cells. ICOS expression helps $\mathrm{T}_{\mathrm{FH}}$ cells maintain their characteristics by blocking the upregulation of klf2, which reverts the $T_{F H}$ phenotype. ${ }^{1}$ Hence, while early blockade of CD28-mediated costimulation reduces $\mathrm{T}_{\mathrm{FH}}$ cell differentiation, prolonged inhibition of CD28-CD80/86 crosstalk by CTLA-4Ig suppresses ICOS signaling and reverts the $\mathrm{T}_{\mathrm{FH}}$ phenotype. Notably, reduced ICOS but also PD-1 expression on $\mathrm{T}_{\mathrm{FH}}$ cells, as reported by Edner et al., can also affect their migratory capacity. ${ }^{14}$ The reduction in $\operatorname{ICOS}^{+} \mathrm{T}_{\mathrm{FH}}$ cell "quantity" in abatacept-treated T1D patients may be due to deactivation and dedifferentiation of $\mathrm{T}_{\mathrm{FH}}$ cells rather than their depletion in the blood. Deactivation and dedifferentiation of $\mathrm{T}_{\mathrm{FH}}$ 
cells may be related to two mechanisms (Fig. 1B), as follows. (1) By inhibiting the crosstalk between the costimulatory molecules CD28 and CD80/CD86, abatacept (CTLA-4lg) might alter $\mathrm{T}_{\mathrm{FH}}$ cell differentiation at two "decision" points in the T-cell zone and the germinal center of secondary lymphoid organs. (2) Abatacept might interfere with CD28-CD80/86-mediated activation of CD4 T cells by DCs at the T-cell zone or the CD4 T-cell interaction with $B$ cells at the T:B cell border, consequently inhibiting $T_{F H}$ cell differentiation, ICOS upregulation, and entry into the $B$ cell follicle. Once in the $B$ cell follicle and the germinal center, abatacept might neutralize CD28-CD80/CD86 interaction among $\mathrm{T}_{\mathrm{FH}}$ cells, GC B cells, and follicular DCs (FDCs), further weakening $T_{F H}$ cell differentiation and proliferation. Interestingly, using whole-blood RNA sequencing, Linsley et al. ${ }^{15}$ identified changes in expression of $B$ cell genes associated with clinical response in individuals with T1D treated with abatacept, thus reinforcing the idea that blocking CD28-CD80/86 interaction may impact GC B cell maturation by reducing $\mathrm{T}_{\mathrm{FH}}$ cell differentiation and activation. In line with this idea, abatacept treatment also caused significant reductions in mIAA antibody levels in the treatment group. ${ }^{15}$

Further studies are required to explore the effect of abatacept on $T_{F H}$ cells, including the correlation of $T_{F H}$ cells and $T_{F R}$ cells with $B$ cell function, islet autoantibodies, and other markers of $B$ cell activation. Nevertheless, the work of Edner et al. indicates that exploitation of cutting-edge methodology such as machine learning approches to analyze immunological profiles may be instrumental for highlighting new pathogenic mechanisms in T1D and, most importantly, may allow pathogenesis-based stratification of T1D patients, thus paving the way for precision medicine in T1D.

\section{REFERENCES}

1. Edner, N. M. et al. Follicular helper T cell profiles predict response to costimulation blockade in type 1 diabetes. Nat. Immunol. 21, 1244-1255 (2020).
2. Todd, J. A. Etiology of type 1 diabetes. Immunity 32, 457-467 (2010).

3. Fousteri, G., Ippolito, E., Ahmed, R. \& Hamad, A. R. A. Beta-cell specific autoantibodies: are they just an indicator of type 1 diabetes? Curr. Diabet. Rev. 13, 322-329 (2017).

4. Chamberlain, N. et al. Rituximab does not reset defective early B cell tolerance checkpoints. J. Clin. Invest. 126, 282-287 (2016).

5. Crotty, S. T follicular helper cell biology: a decade of discovery and diseases. Immunity 50, 1132-1148 (2019).

6. Sherry, N. et al. Teplizumab for treatment of type 1 diabetes (Protege study): 1 year results from a randomised, placebo-controlled trial. Lancet 378, 487-497 (2011).

7. Keymeulen, B. et al. Four-year metabolic outcome of a randomised controlled CD3-antibody trial in recent-onset type 1 diabetic patients depends on their age and baseline residual beta cell mass. Diabetologia 53, 614-623 (2010).

8. Orban, T. et al. Co-stimulation modulation with abatacept in patients with recentonset type 1 diabetes: a randomised, double-blind, placebo-controlled trial. Lancet 378, 412-419 (2011).

9. Zhao, J. et al. Immune and genomic correlates of response to anti-PD-1 immunotherapy in glioblastoma. Nat. Med. 25, 462-469 (2019).

10. Rachid, O., Osman, A., Abdi, R. \& Haik, Y. CTLA4-lg (abatacept): a promising investigational drug for use in type 1 diabetes. Expert Opin. Investig. Drugs 29, 221-236 (2020).

11. Ferreira, R. C. et al. IL-21 production by CD4+ effector T cells and frequency of circulating follicular helper $T$ cells are increased in type 1 diabetes patients. Diabetologia 58, 781-790 (2015).

12. Vecchione, A. et al. Reduced PD-1 expression on circulating follicular and conventional FOXP3 $(+)$ Treg cells in children with new onset type 1 diabetes and autoantibody-positive at-risk children. Clin. Immunol. 211, 108319 (2020).

13. McAdam, A. J. et al. Mouse inducible costimulatory molecule (ICOS) expression is enhanced by CD28 costimulation and regulates differentiation of CD4+ T cells. J. Immunol. 165, 5035-5040 (2000).

14. $\mathrm{Xu}, \mathrm{H}$. et al. Follicular T-helper cell recruitment governed by bystander $\mathrm{B}$ cells and ICOS-driven motility. Nature 496, 523-527 (2013).

15. Linsley, P. S., Greenbaum, C. J., Speake, C., Long, S. A. \& Dufort, M. J. B lymphocyte alterations accompany abatacept resistance in new-onset type 1 diabetes. $J C l$ Insight 4, e126136 (2019). 